\title{
Uc.416 + A promotes epithelial-to- mesenchymal transition through miR-153 in renal cell carcinoma
}

\author{
Yohei Sekino ${ }^{1,2}$, Naoya Sakamoto', Keisuke Goto ${ }^{3}$, Ririno Honma', Yoshinori Shigematsu ${ }^{1,2}$, Thang Pham Quoc ${ }^{1}$, \\ Kazuhiro Sentani ${ }^{1}$, Naohide Oue ${ }^{1}$, Jun Teishima ${ }^{2}$, Fumi Kawakami ${ }^{4}$, Jose A Karam ${ }^{5}$, Kanishka Sircar ${ }^{4,6}$, \\ Akio Matsubara ${ }^{2}$ and Wataru Yasui ${ }^{*}$ (i)
}

\begin{abstract}
Background: The transcribed ultraconserved regions (T-UCRs) are a novel class of non-coding RNAs that are absolutely conserved across species and are involved in carcinogenesis in some cancers. However, the expression and biological role of T-UCRs in renal cell carcinoma (RCC) remain poorly understood. This study aimed to examine the expression and functional role of Uc.416 + A and analyze the association between Uc.416 + A and epithelial-tomesenchymal transition in RCC.

Methods: Expression of Uc.416 + A in 35 RCC tissues, corresponding normal kidney tissues and 13 types of normal tissue samples was determined by quantitative reverse transcription-polymerase chain reaction (GRT-PCR). We performed a cell growth and migration assay in RCC cell line 786-O transfected with negative control and siRNA for Uc. $416+$ A. We evaluated the relation between Uc.416 + A and miR-153, which has a complimentary site of Uc.416 + A.

Results: qRT-PCR analysis revealed that the expression of Uc.416 + A was higher in RCC tissues than that in corresponding normal kidney tissues. Inhibition of Uc.416 + A reduced cell growth and cell migration activity. There was an inverse correlation between Uc.416 + A and miR-153. Western blot analysis showed Uc.416 + A modulated Ecadherin, vimentin and snail. The expression of Uc.416 + A was positively associated with the expression of SNAI1, VIM and inversely associated with the expression of $\mathrm{CDH}$.

Conclusions: The expression of Uc.416 + A was upregulated in RCC and especially in RCC tissues with sarcomatoid change. Uc.416 + A promoted epithelial-to-mesenchymal transition through miR-153. These results suggest that Uc.416 + A may be a promising therapeutic target.
\end{abstract}

Keywords: Uc.416 + A, Renal cell carcinoma, miR-153, Epithelial-to-mesenchymal transition, Sarcomatoid change

\section{Background}

Renal cell carcinoma (RCC) accounts for 3\% of adult malignancies worldwide [1]. Although there have been improvements in the early diagnosis and surgical treatment of RCC, approximately $30 \%$ of RCC tumors are already metastatic at initial diagnosis, and $20-30 \%$ of patients who have undergone surgical extirpation will develop distant metastasis [2]. The prognosis of

\footnotetext{
*Correspondence: wyasui@hiroshima-u.ac.jp

${ }^{1}$ Department of Molecular Pathology, Hiroshima University Institute of Biomedical and Health Sciences, 1-2-3 Kasumi, Minami-ku, Hiroshima 734-8551, Japan

Full list of author information is available at the end of the article
}

metastatic RCC is poor in part because RCC is often resistant to traditional therapies, such as radiation therapy and chemotherapy. Metastatic RCC may result from epithelial-to-mesenchymal transition (EMT) [3]. Therefore, identifying new molecular mechanisms underlying EMT represents an area of great clinical significance for metastatic RCC patients.

Recently, reports have shown that noncoding RNAs (ncRNAs) are most likely to be essential regulators of the development and progression of RCC [4-6]. Transcribed ultraconserved regions (T-UCRs) are novel class of ncRNAs which are highly conserved among most of the vertebrate genomes [7]. Additionally, T-UCRs are frequently located

(c) The Author(s). 2018 Open Access This article is distributed under the terms of the Creative Commons Attribution 4.0 International License (http://creativecommons.org/licenses/by/4.0/), which permits unrestricted use, distribution, and 
at both fragile sites and cancer-associated genomic regions [8], indicating that T-UCRs are believed to play critical roles in human cancer. Moreover, it appears that some T-UCRs serve as oncogenes or tumor suppressor genes in some cancers $[9,10]$. To date, the interaction between T-UCRs and microRNAs is well-studied with evidence linking T-UCRs with cancer progression. We previously demonstrated that Uc.416 + A was upregulated in gastric cancer and was downregulated in prostate cancer. Uc.416 + A was directly regulated by miR-153 and promoted cancer progression in gastric cancer [11], indicating that Uc.416 + A plays an essential role in cancer progression in some cancer. However, little is known about the expression and biological role of T-UCRs including Uc.416 + A in RCC.

In the present study, we examined the expression of Uc.416 + A in RCC tissues, investigated its functional role of Uc.416 + A in RCC progression and analyzed its involvement in EMT. The current study is the first to investigate the expression and functional role of Uc.416 + A in RCC.

\section{Methods}

\section{Tissue samples}

We used 35 RCC tissue samples (Table 1) for quantitative reverse transcription-polymerase chain reaction (qRTPCR). The samples were collected from patients at Hiroshima University Hospital or an affiliated hospital. We obtained 15 frozen sarcomatoid RCC tissue samples and adjacent normal kidney samples from the tissue bank of The University of Texas MD Anderson Cancer Center (Houston, TX) after informed consent and using an institutional review board-approved protocol (IRB\# LAB 08-670). The clinicopathologic features of this cohort are summarized in Table 2.

\section{Cell lines}

Caki1 (ATCC ${ }^{\circledast}$ CRL-1611) and 786-O (ATCC ${ }^{\circledast}$ CRL-1932) were derived from human kidney cancer (Sumitomo Pharmaceuticals Company, Tokyo, Japan). Both cell lines were maintained as described previously [12]. Both cell lines were tested for mycoplasma contamination by PCR.

\section{qRT-PCR analysis}

Extraction of total RNA, synthesis of cDNA, and qRT-PCR was performed as described previously [13]. Gene-specific amplification of all primers we used in this study was confirmed by a single peak in melting curve analysis (date not shown).

To quantify the level of microRNAs, TaqMan assays were performed as described previously [14]. The expression values were normalized to the expression of the small RNA gene RNU6. miR-153 and RNU6 (Thermo Fisher Scientific, Waltham, MA) were used. The primer sequences and IDs were summarized in Additional file 1: Table S1.
Table 1 Clinicopathologic characteristics of 35 RCC tissue samples

\begin{tabular}{ll}
\hline Number of cases & 35 \\
\hline Gender & 28 \\
F & 7 \\
Median age (years) & $64(55-86)$ \\
Race & \\
Asian & 35 \\
Histology & \\
Clear cell RCC & 32 \\
Papillary RCC & 3 \\
Pathological T stage & \\
PT1 & $5(14 \%)$ \\
pT2 & $12(34 \%)$ \\
pT3 & $12(34 \%)$ \\
pT4 & $1(3 \%)$ \\
NA & $5(14 \%)$ \\
Pathological N stage & \\
0 & $19(54 \%)$ \\
1 & $11(31 \%)$ \\
NA & $5(14 \%)$ \\
Metastasis at time of diagnosis & \\
0 & $17(49 \%)$ \\
NA & \\
\hline RCC: & \\
\hline & \\
\hline
\end{tabular}

RCC: renal cell carcinoma, $N A$ : not available

\section{RNA interference}

Silencer ${ }^{\circledast}$ Select (Ambion, Austin, TX) against Uc.416 + A was used for RNA interference as described previously [11]. The sequence of siRNA\#1 was 5'-GCAUCGCUAUAAUU CAUUAga-3', and that of siRNA\#2 was 5'-GCAUACAUA GCAAAACGAAac-3'. Transfection of cells was carried out with Lipofectamine RNAiMAX (Invitrogen) according to the manufacturer's instructions.

\section{Cell growth assay and wound healing assay}

To examine cell growth, an MTT assay was performed as described previously [15]. Cell growth was monitored after 1,2 , and 4 days. To examine cell migration, a wound healing assay was performed as described previously [16].

\section{Western blot analysis}

For Western blot analysis, cells were lysed as described previously [17]. Primary antibody. E-cadherin, snail, and vimentin (Cell Signaling Technology, Inc., Danvers, MA) were used. $\beta$-Actin (Sigma-Aldrich, St. Louis, MO) was used as a loading control. The IDs and dilution of primary and secondary antibody were summarized in Additional file 2: Table S2. 
Table 2 Clinicopathologic characteristics of 15 RCC tissue samples with sarcomatoid change

\begin{tabular}{ll}
\hline Number of cases & 15 \\
\hline Gender & 7 \\
M & 8 \\
Median age (years) & $62(38-76)$ \\
Race & \\
White & 11 \\
Hispanic & 3 \\
Asian & 1 \\
Histology & \\
Clear cell RCC & 11 \\
Papillary RCC & 3 \\
Clear cell papillary RCC & 1 \\
Mean sarcomatoid component (\%) & $38(5-90 \%)$ \\
Pathological T stage & \\
pT1 & $1(6.6 \%)$ \\
pT3 & $13(86.6 \%)$ \\
pT4 & $1(6.6 \%)$ \\
Pathological N stage & $5(33.3 \%)$ \\
0 & $10(66.6 \%)$ \\
\hline Not available & $5(33.3 \%)$ \\
Metastasis at time of diagnosis & $5.3 \%)$ \\
\hline RCC: & \\
\hline & \\
\hline &
\end{tabular}

$R C C$ renal cell carcinoma

\section{Transfection of miR-153 mimics}

miR-153 mimics (Thermo Fisher Scientific, Waltham, MA) were used for the transfection of miR-153 as described previously [11]. Transfection was performed with Lipofectamine RNAiMAX (Invitrogen) according to the manufacturer's protocol.

\section{Luciferase reporter analysis}

The sequence of Uc.416 + A which was complementary sequence of miR-153 was subcoloned into the downstream of the luciferase reporter gene in the pGL3-Promoter vector as previously described [11]. The mutant sequence was generated using primer STAR Mutagenesis Basal Kit (TAKARA BIO, Shiga, Japan). For the reporter assay, 786-O cells were plated onto 24-well plates and transfected with pGL3, pGL3-wild Uc.416 + A, or pGL3-Uc.416 + A -mut vector and miR-153 mimics or negative control using Lipofectamine RNAiMAX (Invitrogen). A Renilla luciferase vector pGL 4.75 (Promega Corporation) was co-transfected in order to normalize the differences in transfection efficiency. Luciferase activity was evaluated consequently via
Dual-GLO Luciferase Assay System (Promega) following producer's manual.

\section{Statistical analysis}

Paired $\mathrm{T}$ test was used to compare the statistical differences between RCC tissues and their corresponding normal kidney tissues. Statistical differences were evaluated using the two-tailed Student $t$-test or MannWhitney $U$-test. $P$-value of $<0.05$ was considered statistically significant. Statistical analyses were conducted primarily using GraphPad Prism software (GraphPad Software Inc., La Jolla, CA).

\section{Results}

Expression of Uc.416 + A is upregulated in RCC tissues

We compared the expression of Uc. $416+\mathrm{A}$ in $35 \mathrm{RCC}$ tissues and corresponding normal kidney tissues by qRT-PCR and considered a RCC tissue/normal kidney tissue ratio $>2.0$ as upregulation. As shown in Fig. 1a, expression of Uc.416 + A was upregulated in 71\% (25/ $35)$ of RCC tissues compared with the corresponding normal kidney tissues $(P=0.008)$. Notably, the expression of Uc.416 + A was higher in metastatic RCC tissues than that in non-metastatic RCC tissues $(P=0.007)$ (Fig. 1b). Then, we examined the expression of Uc.416 + A in 13 types of normal tissue samples and 35 RCC tissues by qRT-PCR. Among these normal tissue samples, the highest expression of Uc.416 + A was found in the pancreas. As expected, 54\% (19/35) of the RCC tissues showed higher expression of Uc. $416+\mathrm{A}$ than that in the pancreas (Fig. 1c).

\section{Knockdown of Uc.416 + A inhibits RCC cell growth and cell migration}

To further validate the aforementioned findings, we examined the functional role of Uc.416 + A in RCC. We investigated the effects of the downregulation of Uc.416 + A on cell growth and cell migration in 786-O cells showing high Uc.416 + A expression (Fig. 2a). We used siRNA that was specifically designed to target Uc.416 + A and confirmed that the expression of Uc.416 + A was substantially suppressed by treatment with the siRNAs (Fig. 2b). Next, we performed a 4,5-dimethylthiazol-2-yl-2,5-diphenyltetrazolium bromide (MTT) assay and wound healing assay. Knockdown of Uc.416 + A reduced cell growth and cell migration activity (Fig. 2c, d, Additional file 3: Figure S1).

\section{Interaction between Uc.416 + A and miR-153}

Several recent evidence has shown that the interaction between T-UCRs and microRNAs plays essential roles in cancer biology $[18,13]$. Previously, we have reported that miR-153 directly regulates Uc. $416+\mathrm{A}$, which contribute to gastric cancer progression via the regulation of tumor cell growth [11]. We therefore investigated the expression 
A

\section{Uc.416+A}

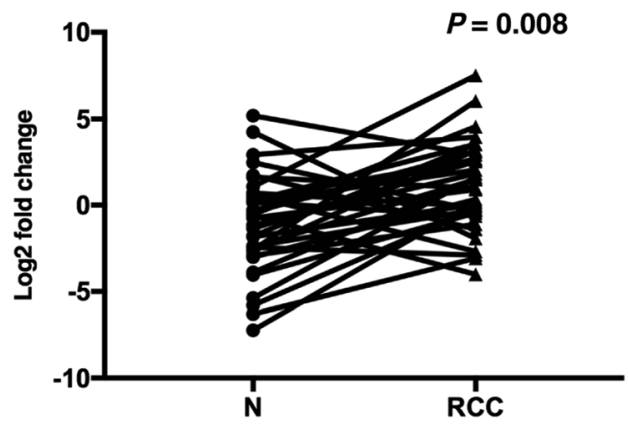

B

Uc.416+A

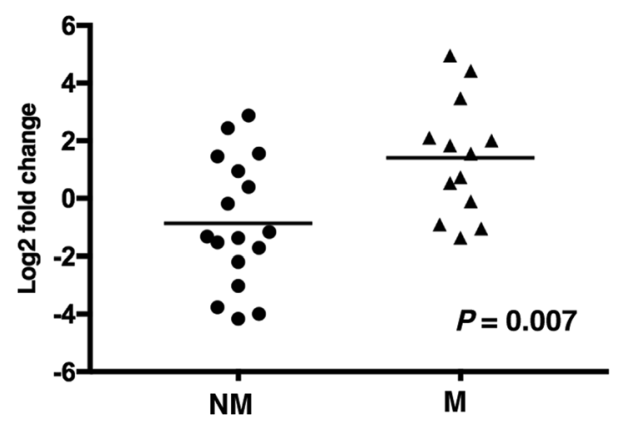

C

Uc.416+A

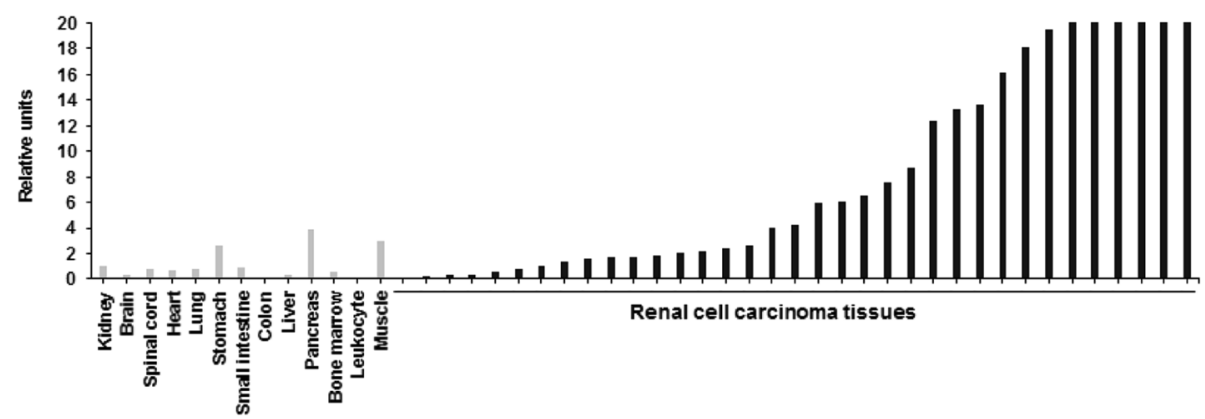

Fig. 1 Overexpression of UC.416 + A in renal cell carcinoma tissues (RCC). a The results of qRT-PCR analysis for the expression of Uc.416+A in 35 RCC tissues and corresponding normal kidney tissues (N). Statistical differences were evaluated with the paired T test. b Scatter plot diagrams showing the association between the expression of Uc.416 + A and metastatic status. Statistical differences were evaluated with the MannWhitney U-test. NM: non-metastatic RCC tissues. M: metastatic RCC tissues. c Results of qRT-PCR analysis for the expression of Uc.416+ A in 35 RCC tissues and 13 types of normal samples

of miR-153 in 32 RCC tissues and corresponding normal kidney tissues by qRT-PCR and considered a RCC tissue/ normal kidney tissue ratio $<1.0$ as downregulation. The expression of miR-153 was downregulated in $75 \%(24 / 32)$ of RCC tissues compared with the corresponding normal kidney tissues $(P=0.011)$ (Fig. 3a). Although correlation coefficient did not reach 0.7 , the expression of miR-153 inversely correlated with the expression of Uc.416 + A $(P=0.002, R=-0.51)$ (Fig. 3b). To further investigate the interaction between Uc.416 + A and miR-153, we examined the effect of Uc.416 + A deregulation on the expression of miR-153. Knockdown of Uc.416 + A upregulated 

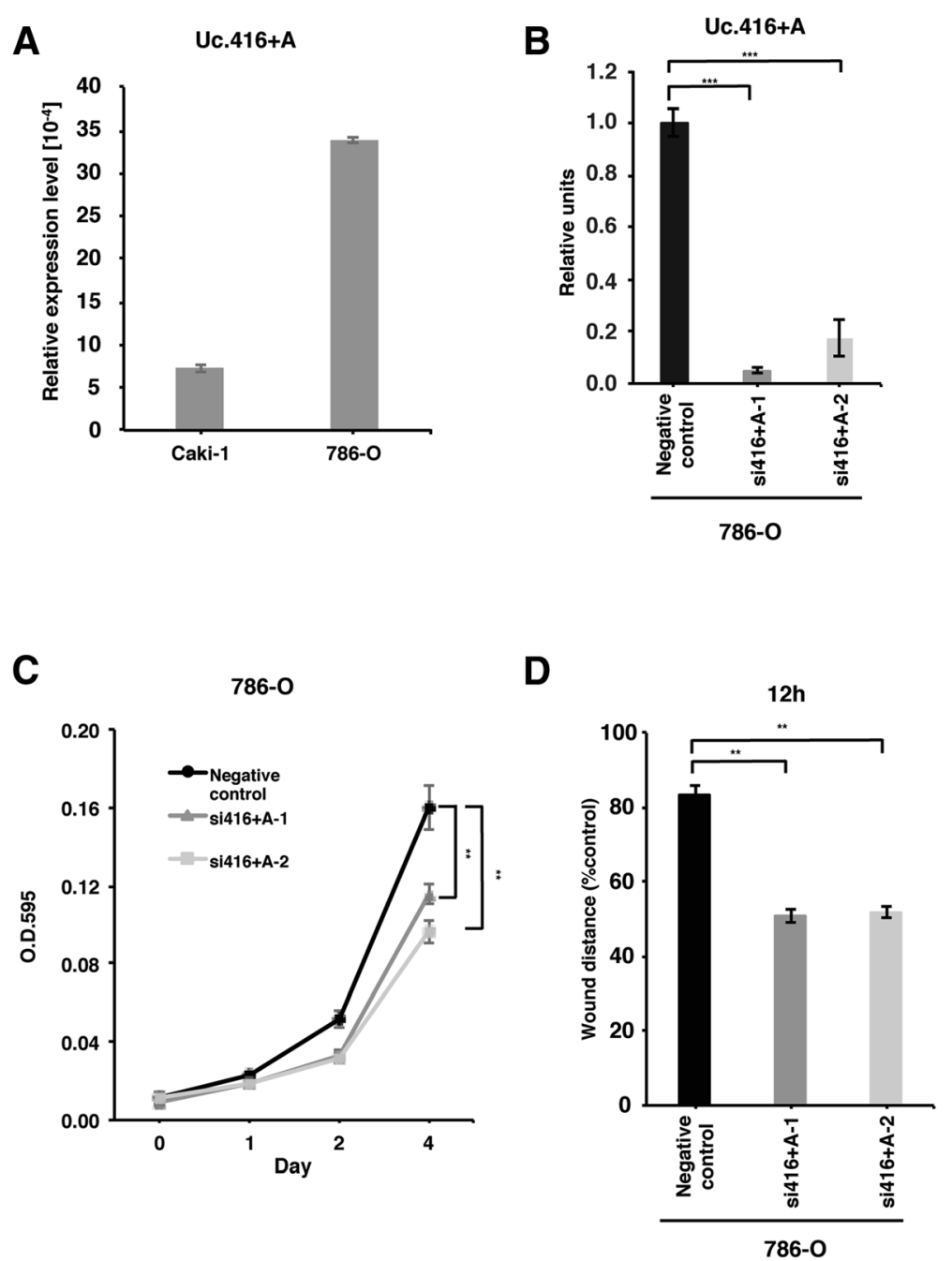

Fig. $2 \mathrm{Uc.416}+\mathrm{A}$ is involved in cell proliferation and migration in 786-O cells. a The results of qRT-PCR analysis for the expression of Uc.416 + A in 786-O and Caki-1 cells. The results are expressed as the mean \pm S.D. of triplicate measurements. $\mathbf{b}$ The results of qRT-PCR analysis for the expression of Uc.416 + A in 786-O cells transfected with negative control or two different siRNAs. The results are expressed as the mean \pm S.D. of triplicate measurements. ${ }^{* *} P<0.001$. c Cell proliferation assay in 786-O cells transfected with negative control or two different siRNAs. Cell growth was assessed by MTT assays at 1, 2, and 4 days after seeding on 96-well plates. Bars and error bars indicate the mean and S.D., respectively, of 3 independent experiments. ${ }^{* *} P<0.01$. $\mathbf{d}$ Wound healing assay in $786-\mathrm{O}$ cells transfected with negative control or two different siRNAs. Wound closures were evaluated by wound contraction percentage and closure time at 0,12 , and $24 \mathrm{~h}$ after scratching. The results are expressed as the mean and S.D. of triplicate measurements. ${ }^{* *} P<0.01$

the expression of miR-153 in 786-O cells (Fig. 3c). In contrast, overexpression of miR-153 reduced the expression of Uc.416 + A (Fig. 3d, e). In order to confirm whether similar direct interaction between miR-153 and Uc.416 + A was also seen in RCC, we performed a dual-luciferase reporter assay. The luciferase reporter assay revealed that co-transfection with pGL3-promoter/wild type Uc.416 + A sequence and miR-153 mimics caused a significant decrease in the luciferase activity compared with the negative control. By contrast, the luciferase activity of the mutant Uc.416 + A showed no significant change compared with that of the negative control (Fig. 3f). Collectively, these results indicate that the expression of Uc.416+
A is directly regulated by miR-153 in RCC, which was consistent with a previous evidence found in gastric cancer [11].

\section{Uc.416 + A stimulates epithelial-to-mesenchymal transition}

Several lines of evidence have shown that miR-153 promotes EMT by directly regulating snail $[19,20]$. To investigate the effect of Uc.416 + A on EMT, we analyzed the expression of snail, vimentin and E-cadherin in 786-O cells transfected with negative control or siRNA for Uc.416 + A by Western blot analysis. Knockdown of Uc.416 + A reduced the expression of snail and vimentin, and contrarily 
A

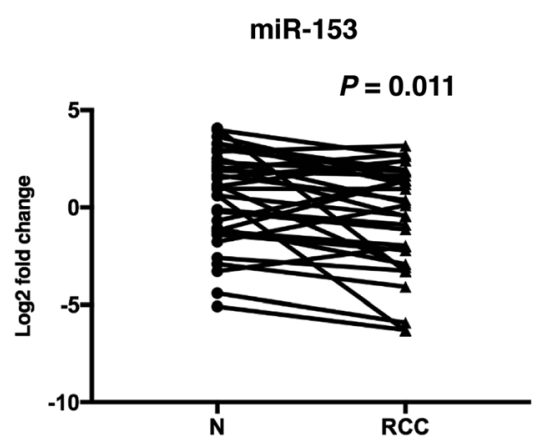

C

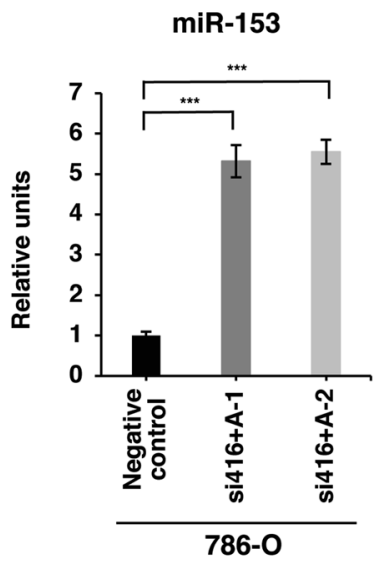

E

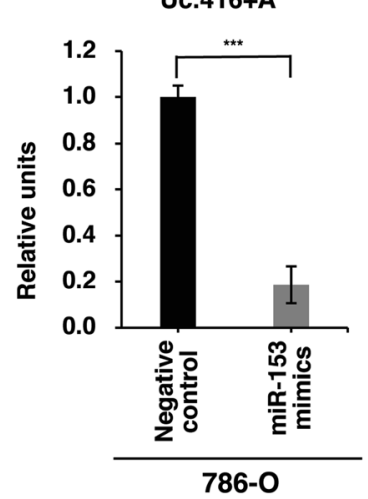

B

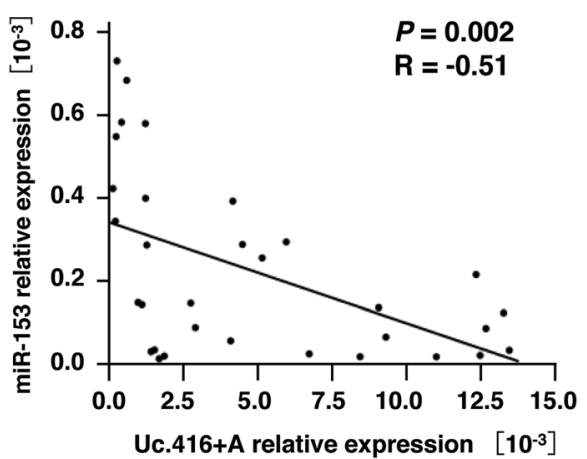

D

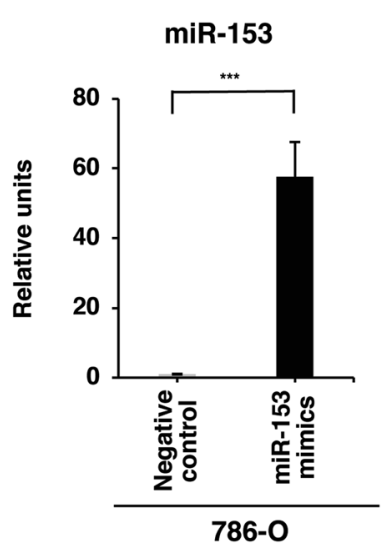

$\mathbf{F}$

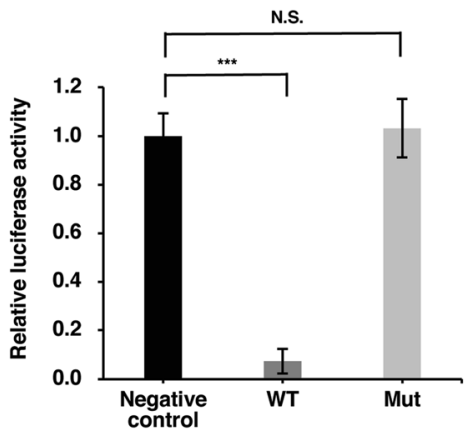

Fig. 3 Interaction between Uc.416 + A and miR-153. a The results of qRT-PCR analysis for the expression of miR-153 in 32 renal cell carcinoma (RCC) tissues and corresponding normal kidney tissues (N). Statistical differences were evaluated with the paired T test. $\mathbf{b}$ The correlation between Uc.416 + A and miR-153 in RCC tissues. Spearman correlation coefficient and P-values are indicated. c qRT-PCR analysis for the expression of miR-153 in 786-O cells transfected with negative control or two different siRNAs. $\mathbf{d}$ qRT-PCR analysis for the expression of miR-153 in 786-O cells transfected with negative control or miR-153 mimics. The results are expressed as the mean and S.D. of triplicate measurements. ${ }^{* * *} P<0.001$. e qRT-PCR analysis for the expression of Uc.416 + A in 786-O cells transfected with negative control or miR-153 mimics. The results are expressed as the mean and S.D. of triplicate measurements. ${ }^{* *} P<0.001$. f The luciferase activity of 786-O cells co-transfected with a control pGL3-Promoter vecor containing a wild type Uc.416 + A sequence or a pGL3-Promoter vector containing a mutated Uc.416 + A sequence. The results are expressed as the mean and S.D. of triplicate measurements. ${ }^{* *} P<0.001$. N.S., not significant; WT: wild type; Mut: sequence with a mutated miR-153 binding site

increased the expression of E-cadherin (Fig. 4a). However, microscopic findings showed that knockdown of Uc.416+ A did not significantly affect cell morphological features (Additional file 4: Figure S2). It has been reported that sarcomatoid change often occurs through EMT [21, 22]. We compared the expression of Uc.416 + A in 15 RCC samples with sarcomatoid change and 35 RCC samples without sarcomatoid change by qRT-PCR. The expression 
A
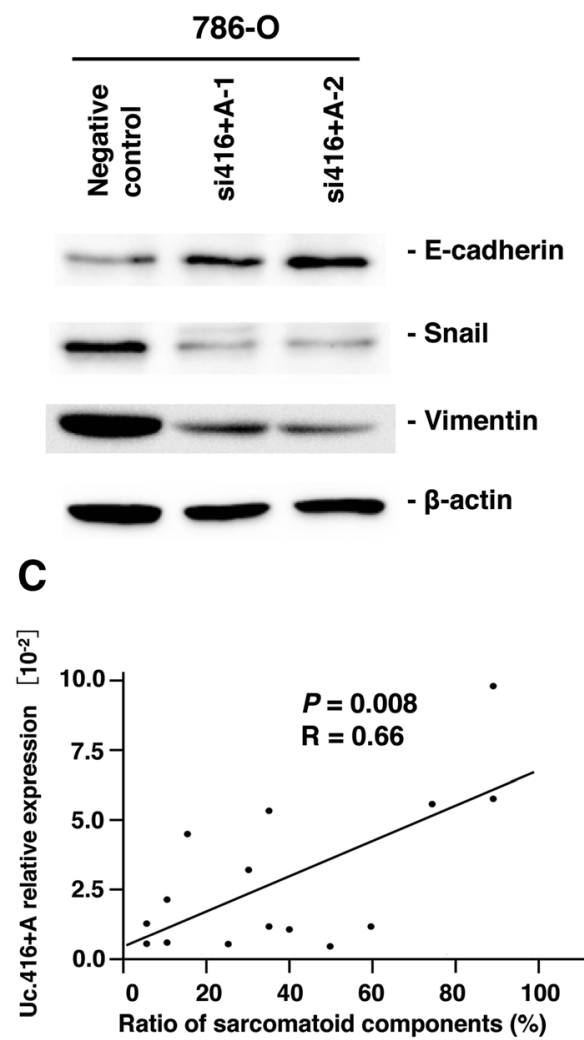

E

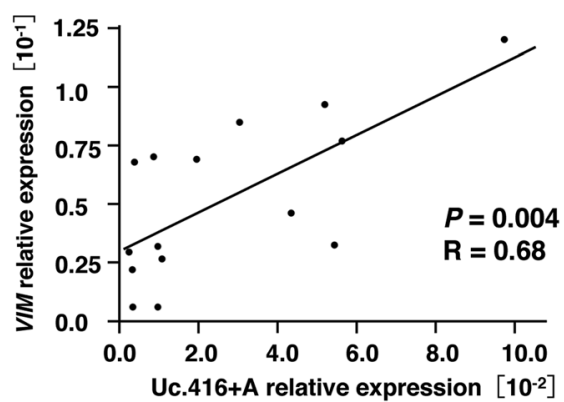

B

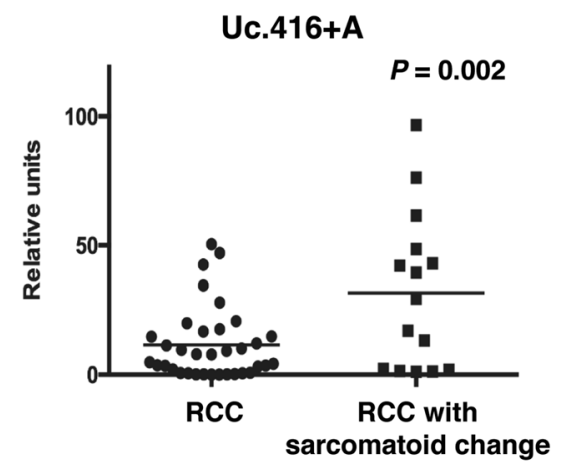

D

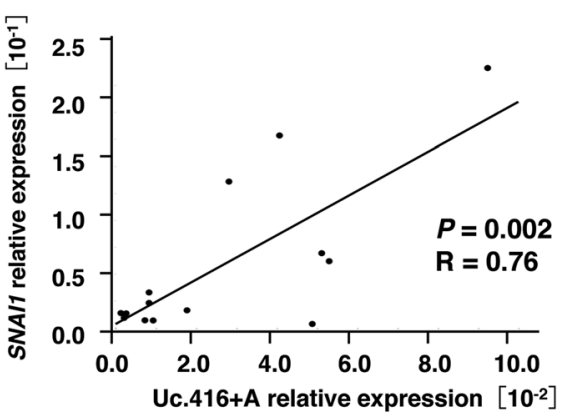

$\mathbf{F}$

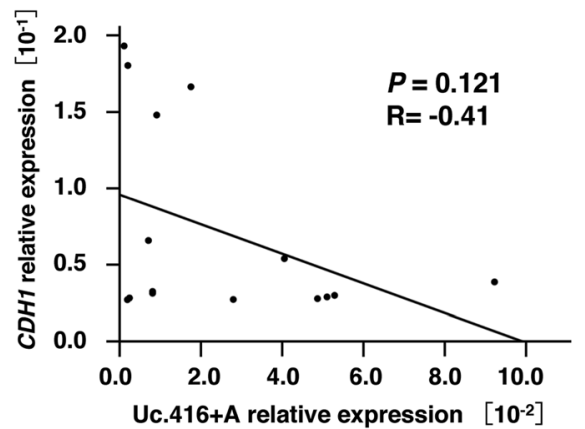

Fig. 4 Uc.416 + A modulates epithelial-to-mesenchymal transition. a Western blot analysis of E-cadherin, snail and vimentin in 786-O cells transfected with negative control or two different siRNAs for Uc.416 + A. $\beta$-actin was used as a loading control. b The relative expression level of Uc.416 + A in 35 RCC samples lacking sarcomatoid change and in 15 RCC samples with sarcomatoid change. $\mathbf{c}$ The correlation between the expression of Uc.416 + A and the ratio of sarcomatoid components in RCC samples with sarcomatoid change. Spearman correlation coefficient and $P$-values are indicated. $\mathbf{d}$ The correlation between the expression of Uc.416 + A and the expression of SNA/1 in RCC samples with sarcomatoid change. Spearman correlation coefficient and $P$-values are indicated. e The correlation between the expression of Uc.416 + A and the expression of VIM in RCC samples with sarcomatoid change. Spearman correlation coefficient and $P$-values are indicated. $\mathbf{f}$ The correlation between the expression of Uc.416 $+\mathrm{A}$ and the expression of $\mathrm{CDH} 1$ in RCC samples with sarcomatoid change. Spearman correlation coefficient and $P$-values are indicated

of Uc.416 + A was higher in the RCC tissues with sarcomatoid change than that in the RCC tissues lacking sarcomatoid change $(P=0.002)$ (Fig. 4b). There was a positive correlation between the expression of Uc.416 + A and the ratio of sarcomatoid components in 15 RCC samples with sarcomatoid change $(P=0.008 R=0.66)$ (Fig. 4c). To further evaluate the relation between Uc.416 + A and EMT, we examined the expression of SNAI1, VIM and CDH1 in RCC samples with sarcomatoid change and corresponding normal kidney tissues by using qRT- 
PCR. The expression of Uc.416 + A correlated positively with that of $\operatorname{SNAII}(P=0.002, R=0.76$, Fig. $4 \mathrm{~d})$ and $\operatorname{VIM}(P=0.004, R=0.68$ Fig. 4e). In contrast, although the differences did not reach statistical significance, the expression of Uc.416 + A correlated inversely with that of $C D H 1(P=0.121, R=-0.41$, Fig. 4f). Taken together, these findings suggest that Uc.416 + A could potentially effect EMT through the regulation of miR-153.

\section{Discussion}

Several studies have shown that non-coding RNAs exhibit aberrant levels of expression in human cancers, and cancer panels based on their expression profiles have been used to distinguish among distinct cancer types [23, 24]. Although recent studies have clearly linked the expression of T-UCRs to cancers $[25,26]$, the systematic characterization of T-UCRs in each cancer is not fully understood. In this study, we observed that Uc.416 + A was upregulated in RCC tissues. Our previous study had shown that Uc.416 + A was upregulated in gastric cancer and downregulated in prostate cancer [11]. Therefore, these findings indicate that the expression of Uc.416 + A varies according to the type of cancer. The development of a wide and robust body of experimental evidence on T-UCRs in cancer may lead to more specific and sensitive cancer panels.

Metastatic RCC is often promoted by reactivating EMT in RCC [3]. During the EMT process, epithelial cells lose their cell-cell adhesion and gain a migratory and invasive mesenchymal phenotype [27]. In this study, Uc.416 + A promoted EMT through the regulation of miR-153, which is the first evidence that EMT can be regulated by a specific T-UCR. We also observed that the expression of Uc.416 + A was higher in RCC tissues with sarcomatoid change than that in RCC tissues lacking sarcomatoid change. Given that EMT is though to play an important role in sarcomatoid differentiation [21], our findings suggest that the promotion of EMT through the interaction between Uc.416 + A and miR-153 may be an important mechanism underlying the EMT process and sarcomatoid differentiation in RCC. As sarcomatoid is known to be resistance to standard therapy and associated with poor overall survival in RCC [28-30], patients are encouraged to participate in clinical trials [31]. In this regard, dysregulated elements leading to EMT may offer promising therapeutic targets. In the present study, we showed that the expression of Uc.416 + A was lower in various normal samples than that in RCC tissues, which implies that Uc.416 + A may be a therapeutic target with fewer adverse effects. Accordingly, these novel findings may improve our understanding of sarcomatoid differentiation and aid in the design of a potential treatment strategy in patients with RCC.

In the present study, we observed that Uc.416 + A promoted EMT through miR-153, which may help to explain how Uc.416 + A contributes to cell migration activity in RCC. However, the mechanism whereby Uc. $416+\mathrm{A}$ is involved in cell growth is not fully understood. To date, several microRNA-T-UCR interactions have been identified that lead to tumorigenesis and cancer progression in some cancers [26, 32]. A recent study has reported that Uc.283 + A inhibited pri-miR-195 processing through direct RNA:RNA interaction [18], which is one of the most plausible machineries between miR-153 and Uc.416 + A. Moreover, a recent study has reported that Uc.338 directly regulated the expression of TIMP-1 and promoted metastasis in colorectal metastasis [33], implying that a messenger RNA (mRNA)-T-UCR interaction played an essential role in the cancer. Based on these findings, there could be unknown interactions among microRNA, mRNA, and T-UCR that are potentially regulated by Uc. $416+$ A. Because the knowledge on T-UCRs as regulators is still in its infancy, further studies are needed to elucidate the regulatory networks related to T-UCRs in cancer.

\section{Conclusion}

Our results showed that Uc.416 + A was overexpressed in RCC, especially in RCC tissues with sarcomatoid change. We also showed that a siRNA target for Uc.416 + A inhibited cell growth and cell migration activity. Furthermore, Uc.416 + A modulated EMT through the regulation of miR-153. Although further studies will be required to clarify how Uc.416 + A contributes to RCC progression, the data presented here highlight the great potential of Uc.416 + A as a therapeutic target in patients with aggressive RCC.

\section{Additional files}

Additional file 1: Table S1. Primers sequence for qRT-PCR. (DOCX 15 kb) Additional file 2: Table S2. ID and dilution of primary and secondary antibody. (DOCX $15 \mathrm{~kb}$ )

Additional file 3: Figure S1. Knockdown of Uc.416 + A reduced cell migration. Representative images of wound healing assays in 786-O cells transfected with negative control or two different siRNAs. (TIF $3180 \mathrm{~kb}$ )

Additional file 4: Figure S2. Knockdown of Uc.416 + A did not significantly affect the morphological features. Representative microscopic findings in 786-O cells transfected with negative control or a siRNA for Uc.416+A. (TIF 4598 kb)

\section{Abbreviations}

EMT: Epithelial-to-mesenchymal; MTT: 4,5-Dimethylthiazol-2-yl)-2,5diphenyltetrazolium bromide; ncRNAs: Noncoding RNAs; qRT-

PCR: Quantitative reverse transcription-polymerase chain reaction; RCC: Renal cell carcinoma; T-UCRs: Transcribed ultraconserved regions

\section{Acknowledgements}

We thank Mr. Shinichi Norimura for his excellent technical assistance. This work was carried out with the kind cooperation of the Research Center for Molecular Medicine of the Faculty of Medicine of Hiroshima University. We also thank the Analysis Center of Life Science of Hiroshima University for the use of their facilities. 


\section{Funding}

This work was supported by Grants-in-Aid for Scientific Research (JP15H04713, JP16K08691) and was mainly supported by Challenging Exploratory Research (26670175, JP16K15247) from the Japan Society for the Promotion of Science.

\section{Availability of data and materials}

All date generated or analyzed during this study are included either in this article or in the supplementary information files. The data that support the findings of this study are available from the corresponding author upon reasonable request.

\section{Author's contributions}

YSe, NS, KG, KS, NO and WY designed the study. YSh, JT, FK, JK, KS and AM provided patients' clinical information. YSe, RH and TQ performed experiments and acquired data. YSh, NS, KG, KS, NO, WY interpreted the results. YSe and NS drafted the manuscript. KS, NO, JT, AM, FK, JK, KS and WY edited it. All Authors approved the final content for journal submission and publication.

\section{Ethics approval and consent to participate}

35 RCC tissue samples were collected from patients at Hiroshima University Hospital or an affiliated hospital under an institutional review boardapproved protocol (IRB\# E912). The written comprehensive informed consent was obtained from all of the patients. This study was conducted in accordance with the Ethical Guidance for Human Genome/Gene Research of the Japanese Government. We also obtained 15 frozen sarcomatoid RCC tissue samples and adjacent normal kidney samples from the tissue bank of The University of Texas MD Anderson Cancer Center (Houston, TX) under approval of the written informed consent and using an institutional review board-approved protocol (IRB\# LAB 08-670).

\section{Consent for publication}

Not applicable.

\section{Competing interests}

The author declare that they have no competing interests.

\section{Publisher's Note}

Springer Nature remains neutral with regard to jurisdictional claims in published maps and institutional affiliations.

\section{Author details}

'Department of Molecular Pathology, Hiroshima University Institute of Biomedical and Health Sciences, 1-2-3 Kasumi, Minami-ku, Hiroshima 734-8551, Japan. ${ }^{2}$ Department of Urology, Hiroshima University Institute of Biomedical and Health Sciences, Hiroshima, Japan. ${ }^{3}$ Cancer Biology Program, University of Hawaii Cancer Center, Honolulu, HI, USA. ${ }^{4}$ Departments of Translational Molecular Pathology, The University of Texas MD Anderson Cancer Center, Houston, TX, USA. ${ }^{5}$ Departments of Urology, The University of Texas MD Anderson Cancer Center, Houston, TX, USA. ${ }^{6}$ Departments of Pathology, The University of Texas MD Anderson Cancer Center, Houston, TX, USA

\section{Received: 25 January 2018 Accepted: 26 September 2018} Published online: 04 October 2018

\section{References}

1. Sandock DS, Seftel AD, Resnick MI. A new protocol for the followup of renal cell carcinoma based on pathological stage. J Urol. 1995;154(1):28-31.

2. Piva F, Giulietti M, Santoni M, Occhipinti G, Scarpelli M, Lopez-Beltran A, Cheng L, Principato G, Montironi R. Epithelial to mesenchymal transition in renal cell carcinoma: implications for Cancer therapy. Mol Diagn Ther. 2016;20(2):111-7.

3. Blondeau JJ, Deng M, Syring I, Schrodter S, Schmidt D, Perner S, Muller SC, Ellinger J. Identification of novel long non-coding RNAs in clear cell renal cell carcinoma. Clin Epigenetics. 2015;7:10.

4. Seles M, Hutterer GC, Kiesslich T, Pummer K, Berindan-Neagoe I, Perakis S, Schwarzenbacher D, Stotz M, Gerger A, Pichler M. Current insights into long non-coding RNAs in renal cell carcinoma. Int J Mol Sci. 2016;17(4):573.

5. Martens-Uzunova ES, Bottcher R, Croce CM, Jenster G, Visakorpi T, Calin GA. Long noncoding RNA in prostate, bladder, and kidney cancer. Eur Urol. 2014;65(6):1140-51.
6. Bejerano G, Pheasant M, Makunin I, Stephen S, Kent WJ, Mattick JS, Haussler D. Ultraconserved elements in the human genome. Science. 2004;304(5675):1321-5.

7. Subramanian A, Tamayo P, Mootha VK, Mukherjee S, Ebert BL, Gillette MA, Paulovich A, Pomeroy SL, Golub TR, Lander ES, et al. Gene set enrichment analysis: a knowledge-based approach for interpreting genome-wide expression profiles. Proc Natl Acad Sci U S A. 2005;102(43):15545-50.

8. Olivieri M, Ferro M, Terreri S, Durso M, Romanelli A, Avitabile C, De Cobelli $\mathrm{O}$, Messere A, Bruzzese D, Vannini I, et al. Long non-coding RNA containing ultraconserved genomic region 8 promotes bladder cancer tumorigenesis. Oncotarget. 2016;7(15):20636-54.

9. Honma R, Goto K, Sakamoto N, Sekino Y, Sentani K, Oue N, Yasui W. Expression and function of Uc.160+, a transcribed ultraconserved region, in gastric cancer. Gastric Cancer. 2017;20(6):960-69.

10. Goto K, Ishikawa S, Honma R, Tanimoto K, Sakamoto N, Sentani K, Oue N, Teishima J, Matsubara A, Yasui W. The transcribed-ultraconserved regions in prostate and gastric cancer: DNA hypermethylation and microRNAassociated regulation. Oncogene. 2016;35(27):3598-606.

11. Pham QT, Oue N, Yamamoto Y, Shigematsu Y, Sekino Y, Sakamoto N, Sentani K, Uraoka N, Tiwari M, Yasui W. The expression of BTS-2 enhances cell growth and invasiveness in renal cell carcinoma. Anticancer Res. 2017;37(6):2853-60.

12. Sekino Y, Sakamoto N, Goto K, Honma R, Shigematsu Y, Sentani K, Oue N, Teishima J, Matsubara A, Yasui W. Transcribed ultraconserved region Uc.63 +promotes resistance to docetaxel through regulation of androgen receptor signaling in prostate cancer. Oncotarget. 2017;8(55):94259-70.

13. Sakamoto N, Naito Y, Oue N, Sentani K, Uraoka N, Zarni Oo H, Yanagihara K, Aoyagi K, Sasaki H, Yasui W. MicroRNA-148a is downregulated in gastric cancer, targets MMP7, and indicates tumor invasiveness and poor prognosis. Cancer Sci. 2014;105(2):236-43.

14. Oue N, Naito Y, Hayashi T, Takigahira M, Kawano-Nagatsuma A, Sentani K, Sakamoto N, Zarni Oo H, Uraoka N, Yanagihara K, et al. Signal peptidase complex 18, encoded by SEC11A, contributes to progression via TGF-alpha secretion in gastric cancer. Oncogene. 2014;33(30):3918-26.

15. Shinmei S, Sentani K, Hayashi T, Sakamoto N, Goto K, Zarni Oo H, Naito Y, Teishima J, Matsubara A, Oue N, et al. Identification of PRL1 as a nove diagnostic and therapeutic target for castration-resistant prostate cancer by the Escherichia coli ampicillin secretion trap (CAST) method. Urol Oncol. 2014;32(6):769-78.

16. Oue N, Hamai Y, Mitani Y, Matsumura S, Oshimo Y, Aung PP, Kuraoka K, Nakayama H, Yasui W. Gene expression profile of gastric carcinoma: identification of genes and tags potentially involved in invasion, metastasis, and carcinogenesis by serial analysis of gene expression. Cancer Res. 2004; 64(7):2397-405

17. Liz J, Portela A, Soler M, Gomez A, Ling H, Michlewski G, Calin GA, Guil S, Esteller M. Regulation of pri-miRNA processing by a long noncoding RNA transcribed from an ultraconserved region. Mol Cell. 2014;55(1):138-47.

18. Wang Z, Liu C. MiR-153 regulates metastases of gastric cancer through snail. Tumour Biol. 2015;37(12):15509-15.

19. Zuo J, Wang D, Shen H, Liu F, Han J, Zhang X. MicroRNA-153 inhibits tumor progression in esophageal squamous cell carcinoma by targeting SNAl1. Tumour Biol. 2016:37(12):16135-40.

20. Conant JL, Peng Z, Evans MF, Naud S, Cooper K. Sarcomatoid renal cell carcinoma is an example of epithelial--mesenchymal transition. J Clin Pathol. 2011;64(12):1088-92.

21. Bostrom AK, Moller C, Nilsson E, Elfving P, Axelson H, Johansson ME. Sarcomatoid conversion of clear cell renal cell carcinoma in relation to epithelial-to-mesenchymal transition. Hum Pathol. 2012;43(5):708-19.

22. Du Z, Fei T, Verhaak RG, Su Z, Zhang Y, Brown M, Chen Y, Liu XS. Integrative genomic analyses reveal clinically relevant long noncoding RNAs in human cancer. Nat Struct Mol Biol. 2013;20(7):908-13.

23. Fabris L, Calin GA. Understanding the genomic Ultraconservations: T-UCRs and Cancer. Int Rev Cell Mol Biol. 2017:333:159-72.

24. Marini A, Lena AM, Panatta E, Ivan C, Han L, Liang H, AnnicchiaricoPetruzzelli M, Di Daniele N, Calin GA, Candi E, et al. Ultraconserved long non-coding RNA uc.63 in breast cancer. Oncotarget. 2016;8(22):35669-80.

25. Calin GA, Liu CG, Ferracin M, Hyslop T, Spizzo R, Sevignani C, Fabbri M, Cimmino A, Lee EJ, Wojcik SE, et al. Ultraconserved regions encoding ncRNAs are altered in human leukemias and carcinomas. Cancer Cell. 2007: 12(3):215-29.

26. He H, Magi-Galluzzi C. Epithelial-to-mesenchymal transition in renal neoplasms. Adv Anat Pathol. 2014;21(3):174-80. 
27. de Peralta-Venturina $M$, Moch H, Amin M, Tamboli P, Hailemariam S, Mihatsch M, Javidan J, Stricker H, Ro JY, Amin MB. Sarcomatoid differentiation in renal cell carcinoma: a study of 101 cases. Am J Surg Pathol. 2001;25(3):275-84.

28. Cheville JC, Lohse CM, Zincke H, Weaver AL, Leibovich BC, Frank I, Blute ML. Sarcomatoid renal cell carcinoma: an examination of underlying histologic subtype and an analysis of associations with patient outcome. Am J Surg Pathol. 2004;28(4):435-41.

29. Shuch B, Bratslavsky G, Linehan WM, Srinivasan R. Sarcomatoid renal cell carcinoma: a comprehensive review of the biology and current treatment strategies. Oncologist. 2012;17(1):46-54.

30. Michaelson MD, McKay RR, Werner L, Atkins MB, Van Allen EM, Olivier KM, Song J, Signoretti S, McDermott DF, Choueiri TK. Phase 2 trial of sunitinib and gemcitabine in patients with sarcomatoid and/or poor-risk metastatic renal cell carcinoma. Cancer. 2015:121(19):3435-43.

31. Peng JC, Shen J, Ran ZH. Transcribed ultraconserved region in human cancers. RNA Biol. 2013;10(12):1771-7.

32. Wang C, Wang Z, Zhou J, Liu S, Wu C, Huang C, Ding Y. TUC.338 promotes invasion and metastasis in colorectal cancer. Int J Cancer. 2017;140(6):1457-64.

33. Chow WH, Dong LM, Devesa SS. Epidemiology and risk factors for kidney cancer. Nat Rev Urol. 2010;7(5):245-57.

Ready to submit your research? Choose BMC and benefit from:

- fast, convenient online submission

- thorough peer review by experienced researchers in your field

- rapid publication on acceptance

- support for research data, including large and complex data types

- gold Open Access which fosters wider collaboration and increased citations

- maximum visibility for your research: over $100 \mathrm{M}$ website views per year

At $\mathrm{BMC}$, research is always in progress.

Learn more biomedcentral.com/submissions 